\title{
Solvent Extraction of Lanthanoid Elements by High Molecular Weight $\beta$-Diketones
}

\author{
Shigeto Nakamura, Yosyong Surakitbanharn ${ }^{\dagger}$ and Kenichi Akiba \\ Research Institute of Mineral Dressing and Metallurgy, Tohoku University, Aoba, Sendai 980, Japan
}

\begin{abstract}
The extraction behavior of lanthanoid elements has been investigated using such $\beta$-diketone derivatives $(H A)$ as dodecylbenzoyltrifluoroacetone (LIX 51), benzoylisooctanoylmethane (LIX 54) and dibenzoylmethane (DBM) in kerosene. Trivalent lanthanoid ions $\left(\mathrm{M}^{3+}\right)$ were extracted as tris- $\beta$-diketonate $\left(\mathrm{MA}_{3}\right)$; the extraction tendency followed the sequence LIX 51》LIX 54>DBM. The extraction constants $\left(K_{\text {ex }}\right)$ for lanthanoid elements increased with an increase in their atomic number, and a "tetrad effect" was approximately observed for each extraction system. The mean separation factor between adjacent elements was evaluated to be 1.71 for LIX 51, 1.95 for LIX 54 and 2.23 for DBM.
\end{abstract}

Keywords High molecular weight $\beta$-diketones, lanthanoid, extraction constant, separation factor, tetrad effect

A number of $\beta$-diketones have been widely used for the extraction of various metal ions in both separation and concentration processes. The extraction of chemically similar rare earths with $\beta$-diketones has been extensively investigated by using simple $\beta$-diketones, such as acetylacetone ${ }^{1,2}$ and thenoyltrifluoroacetone ${ }^{3}$, and it is well known regarding several extraction systems that the extraction efficiency of lanthanoid elements increases with increasing atomic number. ${ }^{1-3}$ However, the application of solvent extraction to largescale separation has been limited, since some $\beta$ diketones are only partly soluble in the aqueous phase and are unstable in the alkaline $\mathrm{pH}$ range. ${ }^{4} \mathrm{~A}$ variety of high molecular weight extractants have been introduced for industrial separation, while reducing extractant loss due to solubility in water. ${ }^{5,6}$ On the other hand, the liquid-liquid extraction method has been extended to convenient solid-liquid extraction for the separation and concentration of metals from dilute solutions by impregnating extractants in inert solid supports, such as membranes and resins. ${ }^{6,7}$ In these cases, selective extracting reagents with restricted solubility in the aqueous phase are required, since these extractants, when impregnated on solid materials, must come into contact with large volumes of aqueous solutions. High molecular weight extractants can also serve as selective reagents in such solid-liquid type extractions, that is, as mobile carriers in liquid membranes and complexing reagents sorbed on macroporous resins.

† On study leave from the Metallurgy Division, Department of Mineral Resources, Ministry of Industry, Bangkok, 10400, Thailand.
The present paper deals with the extraction behavior of lanthanoid by aromatic $\beta$-diketones having hydrophobic groups; ${ }^{8}$ the extraction constants and separation factors have been determined for lanthanoid elements.

\section{Experimental}

\section{Materials}

Solutions of dodecylbenzoyltrifluoroacetone (LIX 51) and benzoylisooctanoylmethane (LIX 54) were obtained from Henkel Corporation and distilled under a reduced pressure of about $2 \mathrm{mmHg}$; the fraction at $169^{\circ} \mathrm{C}$ for LIX 51 was used, as was that at $147^{\circ} \mathrm{C}$ for LIX 54. The concentrations of these reagents were determined by titration with a standard solution of sodium hydroxide in ethanol. Dibenzoylmethane (DBM) purchased from Wako Pure Chemical Ind., Ltd. as a guaranteed reagent was used without further purification.

Radioisotopes, ${ }^{152,4} \mathrm{Eu}$, were purchased from the Radiochemical Centre (England). ${ }^{168} \mathrm{Tm}$ was produced by $30-60 \mathrm{MeV}$ bremsstrahlung irradiation of thulium oxide $(99.99 \%$ purity) with a linear electron accelerator of Tohoku University.

\section{Procedure}

For tracer work, an aqueous solution containing ${ }^{152,4} \mathrm{Eu}(\mathrm{III})$ or about $10^{-5} \mathrm{M}\left(\mathrm{M}=\mathrm{mol} \mathrm{dm}^{-3}\right) \mathrm{Tm}$ (III) spiked with ${ }^{168} \mathrm{Tm}$, was equilibrated by shaking for over $1 \mathrm{~h}$ with an equal volume of a kerosene solution of $\beta$ diketone at $25^{\circ} \mathrm{C}$. The $\mathrm{pH}$ values were controlled with buffer solutions containing acetic acid in the $\mathrm{pH}$ region 
from 4 to 6 and piperazine- $N, N^{\prime}$-bis(2-ethanesulfonic acid) at a pH between 6 and 8; the ionic strength was adjusted to 0.1 with sodium nitrate. After equilibration the radioactivity of both phases was measured with a NaI(T1) scintillation counter. The extraction equilibrium was confirmed by agreement between the distribution ratios for forward and backward extraction.

Determination by inductively coupled plasma atomic emission spectroscopy

In the extraction of a mixture of lanthanoid elements, an aqueous phase initially contained $7 \times 10^{-6}$ to $7 \times 10^{-5} \mathrm{M}$ of each lanthanoid. After equilibration, the concentrations of lanthanoids remaining in the aqueous phase were determined by inductively coupled plasma atomic emission spectroscopy(ICP-AES; SPS 1200A, Seiko Instrument \& Electronics Ltd.). When the distribution ratios were low, the extracted metals were stripped by contacting them with an equal volume of a 1-M nitric acid solution; they were then determined by the ICP-AES method. The linearity of the calibration curve was confirmed over the concentration range of the analyzed samples by using standard solutions of lanthanoid.

\section{Results and Discussion}

\section{Extraction equilibrium}

In order to confirm the extraction equilibria, tracer work was carried out using radioisotopes of ${ }^{152,4} \mathrm{Eu}$ for a typical middle-group lanthanoid and ${ }^{168} \mathrm{Tm}$ for a representative heavy lanthanoid. The distribution ratios $(D)$ of europium(III) and thulium(III) with three

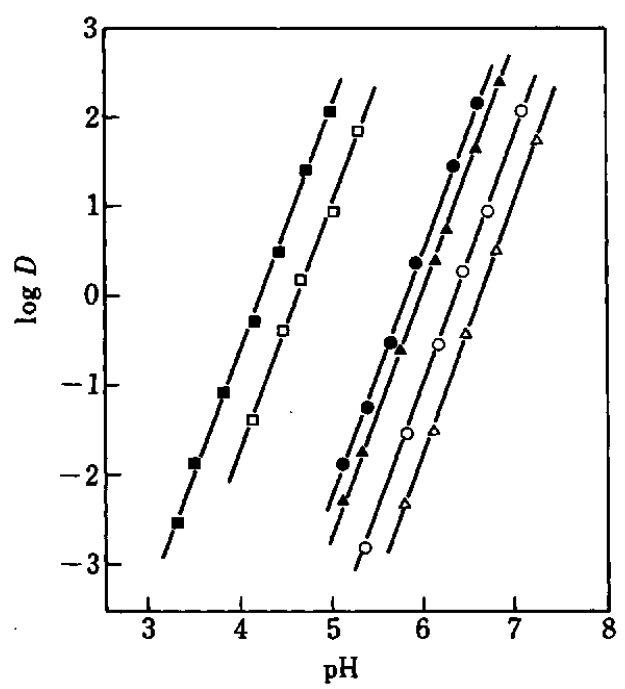

Fig. I Distribution ratios of $\mathrm{Eu}(\mathrm{III})$ and $\mathrm{Tm}$ (III) with $\beta$ diketones in kerosene as a function of $\mathrm{pH}$. $\square \square, 10^{-2} \mathrm{M}$ LIX 51; OO, $10^{-1} \mathrm{M}$ LIX 54; $\triangle \triangle, 10^{-1} \mathrm{M}$ DBM. Open and closed symbols refer to Eu and $\mathrm{Tm}$, respectively. kinds of $\beta$-diketones (LIX 51, LIX 54 and DBM) are shown in Fig. 1, as a function of the pH. Each of these plots is a straight line with a slope close to three. The distribution ratio of a heavier lanthanoid, thulium, is considerably greater than that of europium for each $\beta$ diketone. Figure 2 shows the effect of the concentration of individual extractants on the distribution ratio of europium at fixed $\mathrm{pH}$ values. The distribution ratios are approximately proportional to the third power of the concentration of the $\beta$-diketones, although some

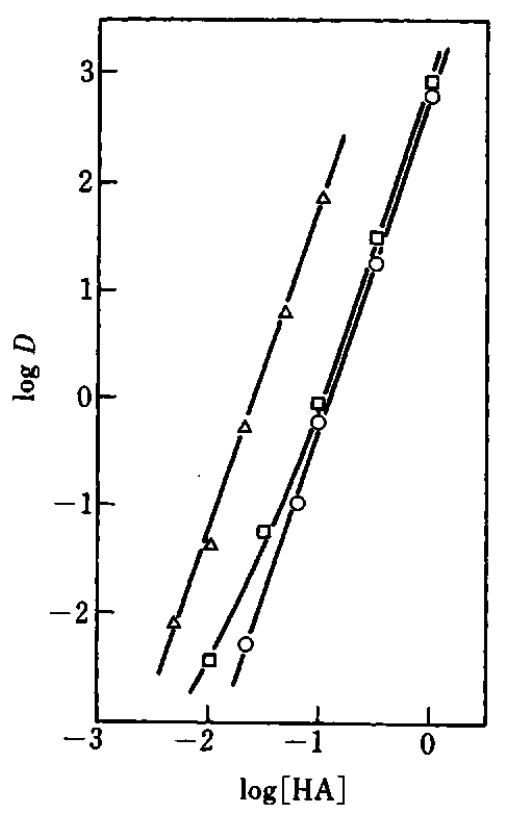

Fig. 2 Distribution ratios of $E u(I I I)$ as a function of $\beta$ diketone concentration. $\square$, LIX 51, pH 3.50; O, LIX 54, pH 6.30; $\triangle$, DBM, pH 7.13.

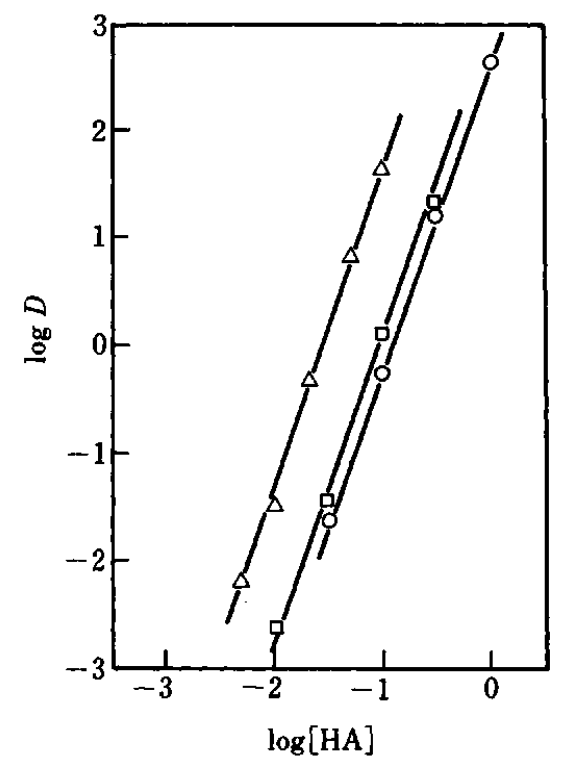

Fig. 3 Distribution ratios of $\operatorname{Tm}(\mathrm{III})$ as a function of $\beta$ diketone concentration. $\square$, LIX 51, pH 3.26; O, LIX 54, pH $5.80 ; \triangle$, DBM, pH 6.54 . 
positive deviations from the line are observed in the low-concentration region of LIX 51. The plots for thulium are also linear, each with a slope of three, as shown in Fig. 3. These findings indicate that the extracted chelate contains three molecules of $\beta$-diketones. Thus, the extraction equilibrium can be expressed as

$$
\mathrm{M}_{\mathrm{aq}}^{3+}+3 \mathrm{HA}_{\mathrm{org}} \rightleftarrows \mathrm{MA}_{3, \text { org }}+3 \mathrm{H}_{\mathrm{aq}}^{+},
$$

where $\mathrm{M}^{3+}$ denotes the lanthanoid ion, HA, $\beta$-diketone and $\mathrm{MA}_{3}$, the extracted chelate. The extraction constant $\left(K_{\text {ex }}\right)$ is given by

$$
K_{\mathrm{ex}}=\frac{\left[\mathrm{MA}_{3}\right]_{\mathrm{org}}\left[\mathrm{H}^{+}\right]_{\mathrm{aq}}^{3}}{\left[\mathrm{M}^{3+}\right]_{\mathrm{aq}}[\mathrm{HA}]_{\mathrm{org}}^{3}} .
$$

The $K_{\text {ex }}$ value evaluated from the experimental data decreased in the order LIX $51 \gg$ LIX $54>$ DBM for both europium and thulium. The highest extraction ability of LIX 51 is probably due to its high acid dissociation constant $\left(\mathrm{p} K_{\mathrm{a}}=6.18\right)^{8}$ induced by the electron-withdrawing effect of the trifluoromethyl group. ${ }^{9}$ A small difference in the $K_{\text {ex }}$ values between LIX 51 and DBM seems to correspond to their similar acidity; that is, $\mathrm{p} K_{\mathrm{a}}=9.64$ and 9.35 , for LIX $54^{8}$ and $\mathrm{DBM}^{10}$, respectively. It was, thus, confirmed from tracer studies for two representative elements that the lanthanoid elements can be effectively extracted by high molecular weight $\beta$-diketones, forming a regular type of $1: 3$ complex $\mathrm{MA}_{3}$ in analogy with simple $\beta$-diketones, such as thenoyltrifluoroacetone; ${ }^{2,3}$ there was no anomalous behavior regarding the present chelate extraction with $\beta$-diketones having a long-chain alkyl group.

\section{Extraction of lanthanoid series elements}

Considerable attention has been given to the chemical properties of lanthanoid and to the separation of these analogous elements. Small differences in the complexing ability are of significant importance for the mutual separation of individual elements by batch and multi-stage techniques. In order to know the difference in the distribution ratios of a series of lanthanoid elements, the extraction behavior of each element was investigated. The distribution ratios between the kerosene solution of LIX 54 and a buffered aqueous solution initially containing a mixture of lanthanoid elements are given in Fig. 4 as a function of the pH. The distribution ratio was determined by measuring the metal concentrations in the aqueous phase by means of ICP-AES. Extraction curves of the lighter lanthanoids with a larger ionic radius show shifts to higher $\mathrm{pH}$ values. Plots of $\log D$ vs. $\mathrm{pH}$ for europium and thulium closely agree with those obtained by the tracer method. These plots, except for cerium, gave parallel lines, each with a slope close to three, indicating that the extraction proceeds via the formation of the 1:3 chelate, $\mathbf{M A}_{3}$. However, cerium appears to be extracted partially as a tetravalent species, according to

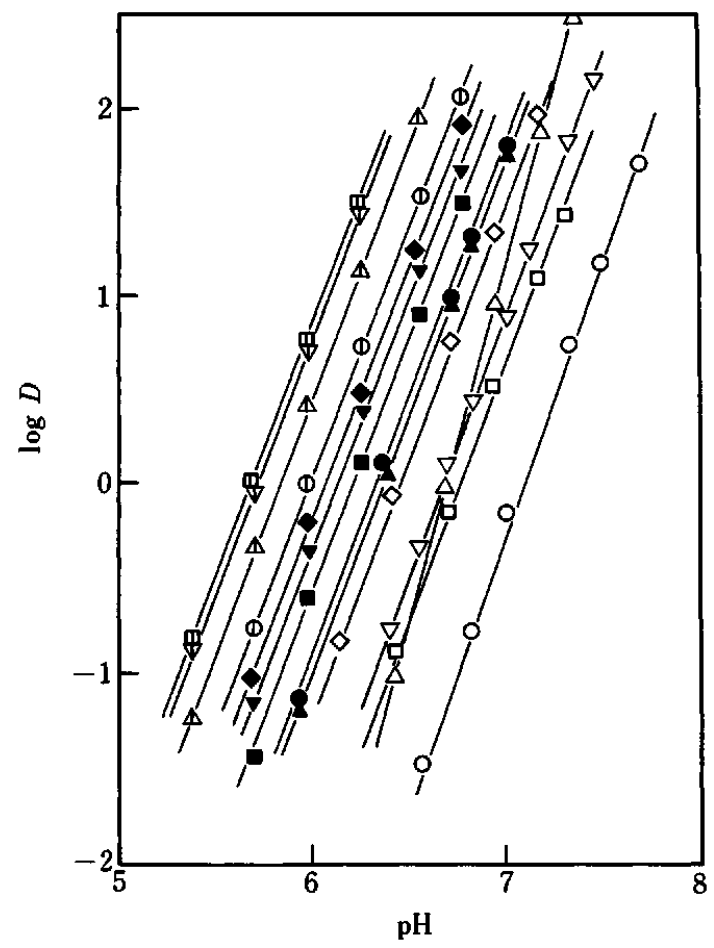

Fig. 4 Extraction of lanthanoids with $10^{-1} \mathrm{M}$ LIX 54 in kero-

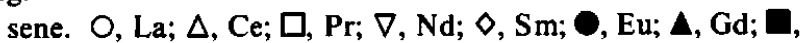

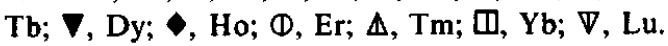

Table 1 Extraction constants $\left(K_{\mathrm{ex}}\right)$ and separation factor $(\alpha)$

\begin{tabular}{|c|c|c|c|c|c|c|}
\hline \multirow{2}{*}{$\begin{array}{l}\text { Lantha- } \\
\text { noids }\end{array}$} & \multicolumn{2}{|c|}{ LIX 51} & \multicolumn{2}{|c|}{ LIX 54} & \multicolumn{2}{|c|}{ DBM } \\
\hline & $\log K_{\mathrm{ex}}$ & $\alpha$ & $\log K_{\mathrm{ex}}$ & $\alpha$ & $\log K_{\mathrm{ex}}$ & $\alpha$ \\
\hline \multirow[t]{2}{*}{${ }_{57} \mathrm{La}$} & -10.54 & & -18.23 & & -19.11 & \\
\hline & & 13.19 & & 9.33 & & 33.11 \\
\hline \multirow[t]{2}{*}{${ }_{59} \mathrm{Pr}$} & -9.41 & & -17.26 & & -17.59 & \\
\hline & & 1.82 & & 1.61 & & 1.17 \\
\hline${ }_{60} \mathrm{Nd}$ & -9.15 & & -17.05 & & -17.52 & \\
\hline \multirow[t]{2}{*}{${ }_{62} \mathrm{Sm}$} & -8.45 & 5.01 & -16.35 & 5.01 & -16.79 & 5.37 \\
\hline & & 1.86 & & 1.55 & & 1.58 \\
\hline \multirow[t]{2}{*}{${ }_{63} \mathrm{Eu}$} & -8.18 & & -16.16 & & -16.59 & \\
\hline & & 0.83 & & 0.98 & & 0.83 \\
\hline \multirow[t]{2}{*}{${ }_{64} \mathrm{Gd}$} & -8.26 & & -16.17 & & -16.67 & \\
\hline & & 1.38 & & 3.72 & & 4.17 \\
\hline \multirow[t]{2}{*}{${ }_{65} \mathrm{~Tb}$} & -8.12 & & -15.60 & & -16.05 & \\
\hline & & 1.41 & & 1.95 & & 2.90 \\
\hline \multirow[t]{2}{*}{${ }_{66} \mathrm{Dy}$} & -7.97 & & -15.31 & & -15.73 & \\
\hline & & 1.09 & & 1.29 & & 1.35 \\
\hline \multirow[t]{2}{*}{${ }_{67} \mathrm{Ho}$} & -7.93 & & -15.20 & & -15.60 & \\
\hline & -7.78 & 1.41 & -14.95 & 1.78 & -15.31 & 1.94 \\
\hline${ }_{68} \mathrm{Er}$ & & 1.91 & & 2.95 & & 2.95 \\
\hline \multirow[t]{2}{*}{${ }_{69} \mathrm{Tm}$} & -7.50 & & -14.48 & & -14.84 & \\
\hline & & 1.74 & & 2.40 & & 2.51 \\
\hline \multirow[t]{2}{*}{${ }_{70} \mathrm{Yb}$} & -7.26 & & -14.10 & & -14.44 & \\
\hline & & 0.93 & & 0.85 & & 1.62 \\
\hline${ }_{71} \mathrm{Lu}$ & -7.29 & & -14.17 & & -14.23 & \\
\hline \multicolumn{2}{|c|}{ Mean value of $\alpha$} & 1.71 & & 1.95 & & 2.23 \\
\hline
\end{tabular}
in lanthanoid- $\beta$-diketone systems

Separation factor $(\alpha)=K_{\mathrm{ex}, \mathrm{A}} / K_{\mathrm{ex}, \mathrm{B}}$. 
its slope, which is close to four.

The extraction constants evaluated from the distribution data are summarized in Table 1. They increase with an increase in the atomic number from ${ }_{57} \mathrm{La}$ to ${ }_{71} \mathrm{Lu}$. This order indicates that the smaller ions are more extracted than the larger ions owing to lanthanoid contraction. The separation factor $(\alpha)$ between elements $A$ and $B$ is given by

$$
\alpha=\frac{D_{\mathrm{A}}}{D_{\mathrm{B}}}=\frac{K_{\mathrm{ex}, \mathrm{A}}}{K_{\mathrm{ex}, \mathrm{B}}}
$$

The values of $\alpha$ are listed in Table 1. The higher separation factors between adjacent elements were obtained for the ${ }_{65} \mathrm{~Tb}-{ }_{64} \mathrm{Gd}$ and ${ }_{69} \mathrm{Tm}-{ }_{68} \mathrm{Er}$ pairs. The average value of the separation factor between the adjacent elements was evaluated to be 1.95 from the difference in the $K_{\mathrm{ex}}$ values of ${ }_{57} \mathrm{La}$ and ${ }_{71} \mathrm{Lu}$.

The distribution ratio in the Eu-DBM system was found to increase slightly with increasing europium concentration over $7 \times 10^{-6} \mathrm{M}$ in a preliminary experiment. Figure 5 shows plots of $\log D$ obtained for a mixture of $7 \times 10^{-6} \mathrm{M}$ of each lanthanoid. These plots show parallel lines, each with a slope of three, indicating the extracted species $\mathrm{MA}_{3}$ (with exception of cerium). The extraction constants given in Table 1 for each element are slightly less than those with LIX 54. Relatively high separation factors were also obtained for pairs of ${ }_{65} \mathrm{~Tb}-{ }_{64} \mathrm{Gd}$ and ${ }_{69} \mathrm{Tm}-{ }_{68} \mathrm{Er}$, and the mean separation factor was found to be 2.23 . The separation efficiency of DBM was slightly lower than that of $\operatorname{di}(2-$ ethylhexyl)phosphoric acid (DEHPA), having an average separation factor of 2.5. ${ }^{11}$

Extraction curves with LIX 51 are illustrated in

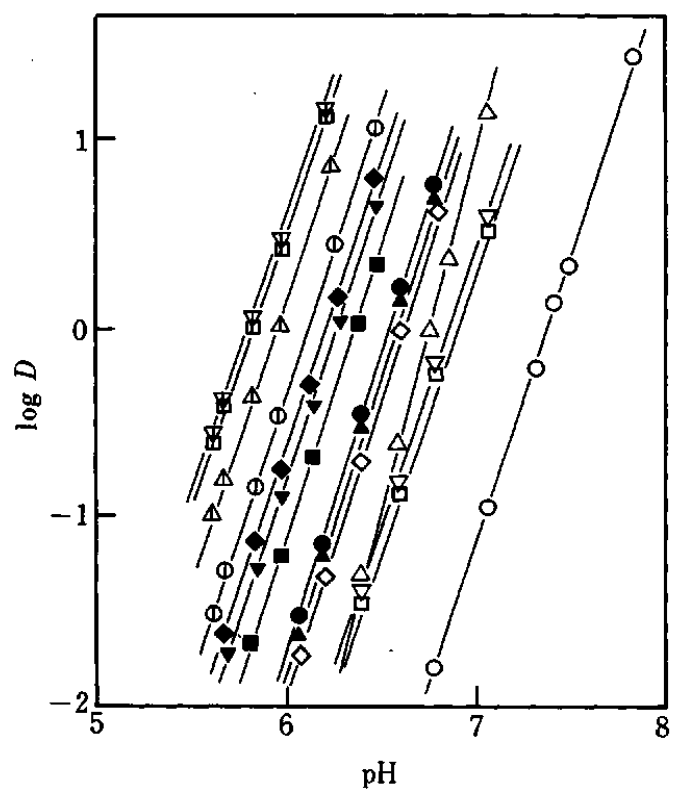

Fig. 5 Extraction of lanthanoids with $10^{-1} \mathrm{M} \mathrm{DBM}$ in kerosene. O, La; $\Delta$, Ce; $\square, \operatorname{Pr} ; \nabla, \mathrm{Nd} ; \diamond, \mathrm{Sm} ; \bigcirc, \mathrm{Eu} ; \mathbf{\Delta}, \mathrm{Gd} ; \mathbf{\square}$,

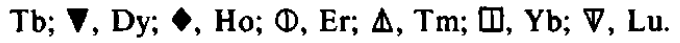

Fig. 6. The distribution ratios were found to decrease with an increase in the metal concentrations; they were determined by employing an aqueous solution initially containing individual ions of $7 \times 10^{-6} \mathrm{M}$, in which the concentration dependence was rather small. The extraction constants of lanthanoids with LIX 51 are about

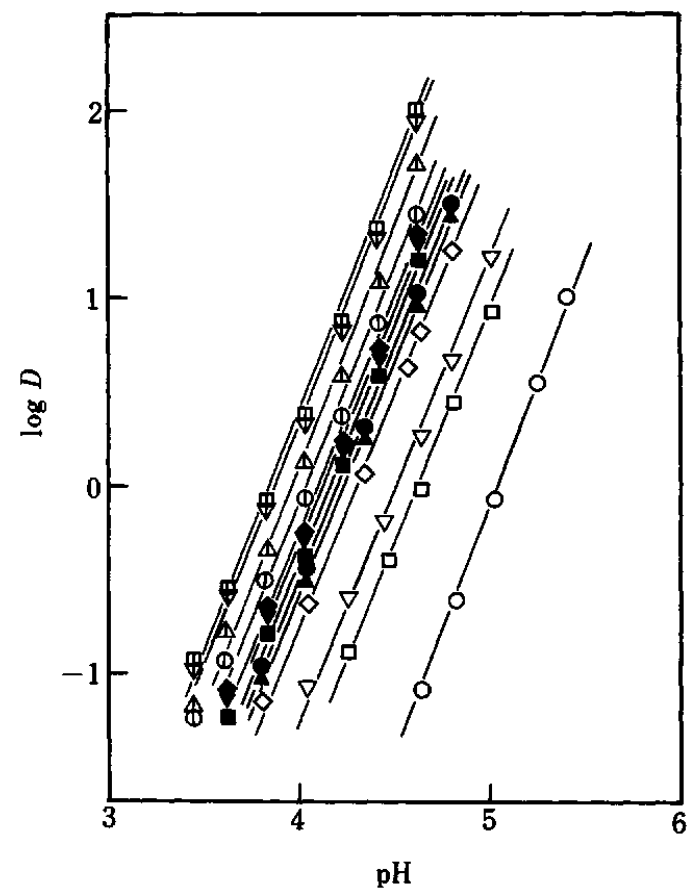

Fig. 6 Extraction of lanthanoids with $3 \times 10^{-2} \mathrm{M}$ LIX 51 in kerosene. O, La; $\square, \operatorname{Pr} ; \nabla, \mathrm{Nd} ; \diamond, \mathrm{Sm} ; \bigcirc, \mathrm{Eu} ; \boldsymbol{\Delta}, \mathrm{Gd} ; \mathbf{\square}, \mathrm{Tb}$;

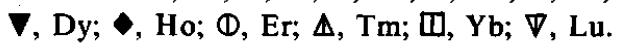

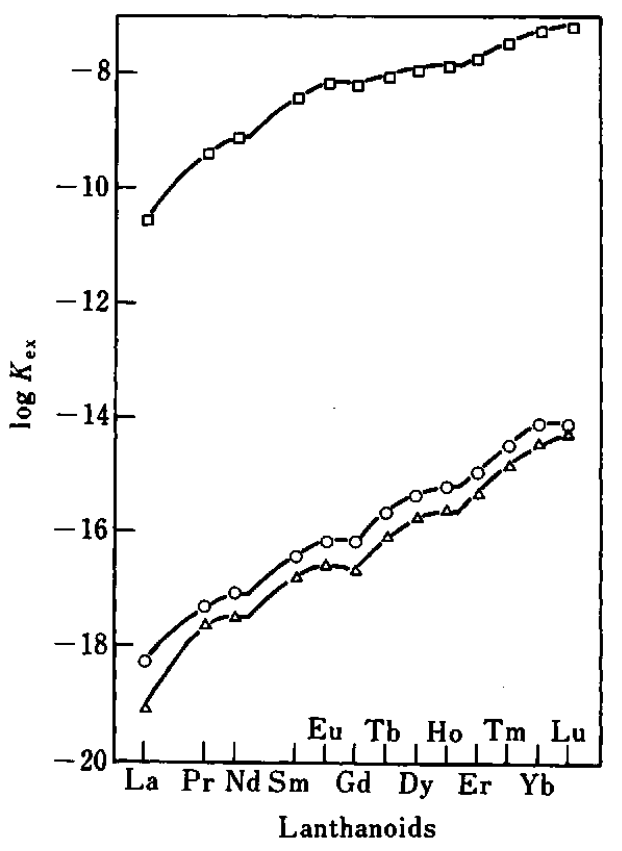

Fig. 7 Variation of the extraction constants with atomic number of lanthanoids. $\square$, LIX 51; O, LIX 54; $\triangle$, DBM. 
eight orders of magnitude larger than those with LIX 54 and with DBM, reflecting the high extractability of LIX 51. The separation factors are somewhat lower compared with those with LIX 54 and DBM; that is, the mean value of $\alpha$ was evaluated as being 1.71. Thus, the extraction constant increased in the order DBM $<$ LIX $54 \ll$ LIX 51, while the separation factor increased in the reverse order, LIX $51<$ LIX $54<$ DBM. These data seem to be a useful guide for the extraction and mutual separation of a series of lanthanoid elements. ${ }^{12}$

\section{Variation of $K_{\text {ex }}$ with atomic number}

Figure 7 illustrates plots of $\log K_{\mathrm{ex}}$ against the atomic number of each lanthanoid element. The $\log K_{\mathrm{ex}}$ values generally increase with increasing atomic number for all $\beta$-diketone systems used. These plots, however, are not linear but, rather, consist of downward concave curves. This trend is similar to a tetrad effect pointed out by Peppard and coworkers regarding certain extraction systems. ${ }^{13}$ Plots of $\log K_{\mathrm{ex}}$ against atomic number would be divided into four tetrad groups: ${ }_{57} \mathrm{La}-$ ${ }_{60} \mathrm{Nd},{ }_{62} \mathrm{Sm}-{ }_{64} \mathrm{Gd},{ }_{64} \mathrm{Gd}-{ }_{67} \mathrm{Ho}$ and ${ }_{68} \mathrm{Er}-{ }_{71} \mathrm{Lu}$. On account of the tetrad concave downward curve, the separation factors between the front elements of a certain tetrad group are relatively high, and those between the rear elements, on the contrary, are lower. As can be seen in Table 1, the separation factors were relatively large for the ${ }_{64} \mathrm{Gd}-65 \mathrm{~Tb}$ and ${ }_{68} \mathrm{Er}-{ }_{69} \mathrm{Tm}$ pairs with LIX 54 and DBM. On the other hand, the separation factors for ${ }_{60} \mathrm{Nd}-{ }_{59} \mathrm{Pr},{ }_{64} \mathrm{Gd}-{ }_{63} \mathrm{Eu},{ }_{67} \mathrm{Ho}-{ }_{66} \mathrm{Dy}$ and ${ }_{71} \mathrm{Lu}-{ }_{70} \mathrm{Yb}$ were rather low compared with the other pairs of adjacent elements. Further, an anomalous order was found for the ${ }_{64} \mathrm{Gd}-63 \mathrm{Eu}$ pair; that is, the $K_{\mathrm{ex}}$ value of ${ }_{64} \mathrm{Gd}$ was slightly less than that of ${ }_{63} \mathrm{Eu}$.

The extractability of LIX 51 was found to be much higher than that of LIX 54 and DBM; however, the difference in $K_{\mathrm{ex}}$ in the series of rare earth elements was rather small. Thus, the tetrad effect is not so clear as that observed in the LIX 54 and DBM systems.
Especially, the separation factors for heavier elements were relatively smaller than those for lighter elements. In these cases the mutual separation of individual elements must be very difficult. The extractability of these $\beta$-diketone extractants are generally weaker than that of dialkylphosphoric acids commonly employed for rare earth extraction. It would, however, be rather preferable for the transport of metal species through a liquid membrane containing these $\beta$-diketones and for the effective recovery of metal ions from $\beta$-diketone impregnating resins.

\section{References}

1. W. B. Brown, J. F. Steinbach and W. F. Wagner, $J$. Inorg. Nucl. Chem., 13, 119 (1960).

2. N. Suzuki and S. Nakamura, Inorg. Chim. Acta, 110, 243 (1985).

3. J. Alstad, I. H. Augustson and L. Farbu, J. Inorg. Nucl. Chem., 36, 899 (1974).

4. A. M. Poskanzer and B. M. Foreman,Jr., J. Inorg. Nucl. Chem., 16, 323 (1961).

5. M. J. Hadson, Hydrometallurgy, 9, 149 (1982).

6. L. L. Tavlarides, J. H. Bae and C. K. Lee, Sep. Sci. Technol., 22, 581 (1987).

7. K. Akiba and H. Hashimoto, Talanta, 32, 824 (1985).

8. H. Watarai, K. Kamada and S. Yokoyama, Sol. Ex. Ion Exch., 7, 361 (1989).

9. T. Honjyo, Bull. Chem. Soc. Jpn., 42, 995 (1969).

10. J. Stary, "The Solvent Extraction of Metal Chelates", Pergamon Press, Oxford, 1964.

11. D. F. Peppard, G. W. Mason, J. L. Maier and W. J. Driscoll, J. Inorg. Nucl. Chem., 4, 334 (1957).

12. K. Akiba, S. Sawai and S. Nakamura, J. Liq. Chromatogr., 11, 2517 (1988).

13. D. F. Peppard, G. W. Mason and S. Lewey, J. Inorg. Nucl. Chem., 31, 2271 (1969).

(Received August 28, 1989) (Accepted September 7, 1989) 